\title{
Formación
}

\section{de maestros,} campo pedagógico y deseo de saber

Teaching training, pedagogy and the known desire
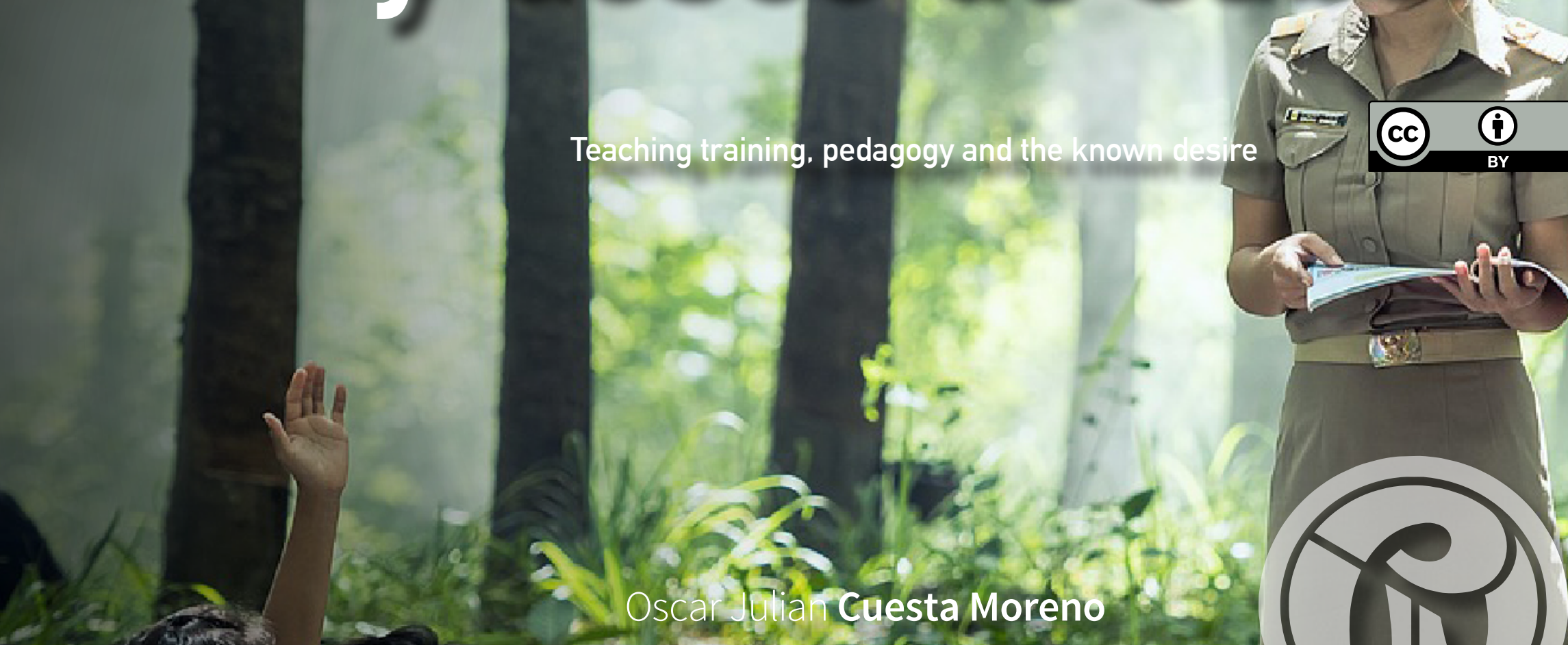

Oscar Julian Cuesta Moreno

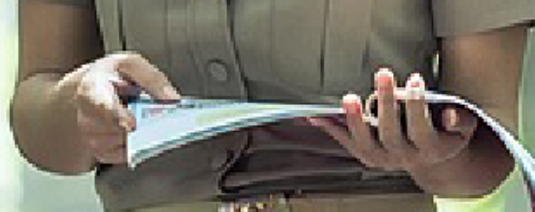




\section{Resumen}

0123-8264.hop.19203

Title:

Teaching training, pedagogy and the known desire

Título: $\quad$ Formación de maestros, campo pedagógico y deseo de saber

Alt Title / Título alternativo:

[en]

Teaching training pedagogical field and desire to know

[es]: $\quad$ Formación de maestros, campo pedagógico y deseo de saber

Author (s) / Autor (es):

Cuesta Moreno

\section{Keywords / Palabras Clave:}

[en]

teacher training; pedagogical field; the intellectual field of education; desire to know

[es]

formación de maestros; campo pedagógico; campo intelectual de la educación; deseo de saber

Proyecto / Project:

Seminario homónimo impartido por el autor en la Maestría en educación de la Universidad Pedagógica Nacional- UPN-Bogotá D.C. Colombia Financiación / Funding:

Formación de maestros; campo pedagógico; campo intelectual de la educación; deseo de saber

Submited: 2017-05-14

Acepted: 2017-07-16

Source I Filiacion:

BIO:

City I Ciudad:

Bogotá DC [Co]

e-mail:
El artículo propone criterios para formar maestros. Para ello, en primera instancia, plantea la necesidad de reconocer el campo conceptual de la pedagogía como una condición fundamental para reivindicar el saber de los maestros y su capacidad de investigar y producir conocimientos. En segundo lugar, expone que la formación de maestros debe darse mediante el encuentro con el saber y el deseo, esto es, llevar al estudiante de las licenciaturas a sumergirse en el campo pedagógico. En tercer lugar, se mostrará la articulación de estos planteamientos con los lineamientos de la Ley General de Educación (Ley 115 de 1994). Finalmente, se propondrá una didáctica para que los estudiantes de las licenciaturas y posgrados en educación logren sumergirse en el campo conceptual de la pedagogía.

\section{Abstract}

The article proposes possible criteria for teacher training. For this, in the first instance, it raises the need to recognize the conceptual field of pedagogy as a fundamental condition to claim the knowledge of teachers and their ability to research and produce new knowledge. Secondly, it states that the training of teachers must take place through the encounter with knowledge and desire, that is, to lead the undergraduate student to immerse himself in the pedagogical field.

Thirdly, it will be shown the articulation of these approaches with the guidelines of the General Law of Education (Act 115 of 1994). Finally, it will propose a didactic so that the students of the bachelor"s and postgraduate degrees in education manage to immerse themselves in the conceptual field of pedagogy.

Oscar Julian Cuesta Moreno, MEd BASC

AutorlD: $\quad 57191375225$

Research ID: G-4920-2015

ORCID: 0000-0001-7181-1183

Universidad Pedagógica Nacional

Magister en Educación, Universidad Pedagógica Nacional

Comunicador Social, Universidad Santo Tomás de Colombia

ojcuestam@pedagogica.edu.co

Citar como:

Cuesta Moreno, O. J. (2017). Formación de maestros, campo pedagógico y deseo de saber. Horizontes Pedagógicos, issn-l:0123-8264, 19 (2), [pgln]-[pgOut]. Obtenido de: https://revistas.iberoamericana.edu.co/index.php/ rhpedagogicos/article/view/1104 


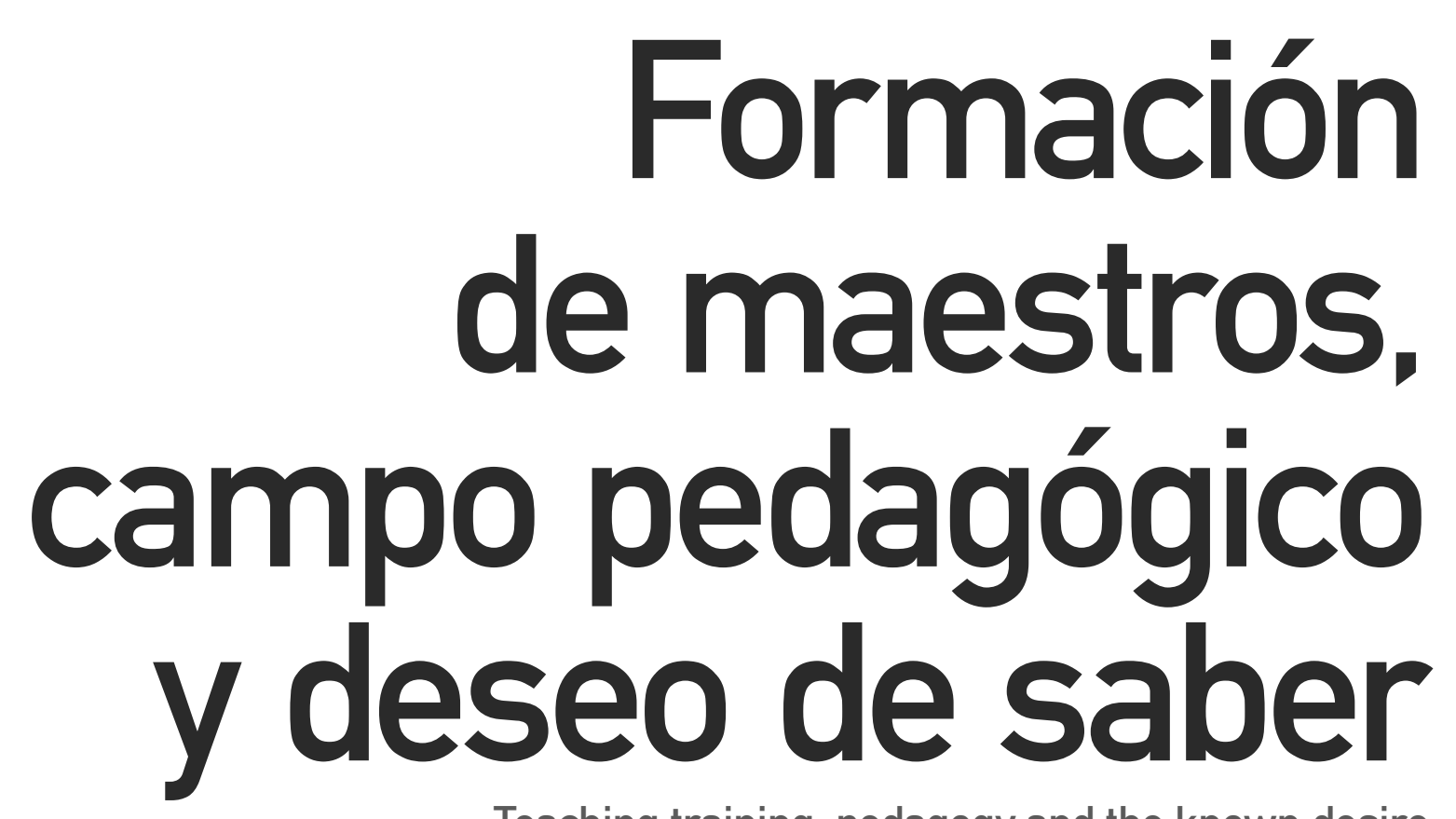

Teaching training, pedagogy and the known desire

Oscar Julian Cuesta Moreno

\section{Excelencia docente}

Las actuales políticas educativas, que centran el éxito de la escolarización en los logros obtenidos por los estudiantes en pruebas estandarizadas, reducen el papel del maestro, dado que van imponiendo contenidos y modos de enseñanza formulados por agentes externos a la escuela y, consecuentemente, reducen también su capacidad analítica y propositiva, obliterando su saber pedagógico.

Estas políticas basadas en estudios econométricos, reconocen en el factor docente un elemento importante de la calidad educativa, sin embargo, siguiendo a Álvarez Gallego (2014) desconocen que el proceso educativo está complejizado por aspectos culturales, económicos, sociales, quenoseresuelven conlaexcelenciadocente. Enefecto, informes como el de la Fundación Compartir (2014), que incide directamente en la agenda pública del Ministerio de Educación, promueven la capacitación y el estímulo salarial a los profesores, pero encubren múltiples aspectos de la realidad educativa.

De hecho, como lo señala Álvarez Gallego (2014), el informe de la Fundación Compartir (2014), desconoce trabajos de autores colombianos quehan investigado, analizadoyteorizadosobreestos múltiples aspectos de la realidad educativa. El desconocimiento de estas investigaciones, no sólo en el informe 'Tras la excelencia docente', sino en general por los agentes que influyen en la política educativa, permite preguntarse por el papel de los intelectuales de la educación colombiana. 
De otro lado, las políticas educativas vigentes, centradas en la profesionalización docente, limitan a los profesores en su capacidad reflexiva, pues valoran más el dominio de estrategias didácticas que lleven a los alumnos a lograr buenos resultados en las pruebas estandarizadas. Es decir, siguiendo a Berstein (1998), el campo de re contextualización oficial, oblitera al campo de recontextualización pedagógico, lo que genera la paulatina pérdida del valor intrínseco al maestro, es decir, el conocimiento pedagógico.

Bajo la lógica de la excelencia docente, formar licenciados sería formar desarrolladores de currículos y estrategas de la enseñanza, pues los contenidos estarían predefinidos, restándole toda la capacidad como sujetos de saber, es decir, eliminando su capacidad de analizar y producir contenidos de acuerdo a los contextos específicos y las particularidades de los estudiantes.

Precisamente, este artículo propone posibles criterios para formar maestros. Por tal razón, en primer lugar, se argumenta que el campo conceptual de la pedagogía es un cimiento fundamental para reivindicar el saber de los maestros. En un segundo momento, el texto sostiene que la formación de maestros deviene del encuentro del deseo con el saber, lo que implica sumergir al estudiante en el campo pedagógico. Esto no es ajeno a la política, por lo que en un tercer momento se expone el vínculo de estos argumentos con la Ley General de Educación (Ley 115 de 1994). Por último, se proyecta una didáctica que logre sumergir a los estudiantes de las licenciaturas y posgrados en educación en el campo conceptual de la pedagogía.

\section{Análisis relacional}

Para formular las tesis y argumentos planteados en el presente texto, se efectuó un ejercicio de análisis relacional, para lo cual se recuperan autores que teorizan sobre el campo conceptual de la pedagogía, el campo intelectual de la educación, la recontextualización en la práctica pedagógica y la formación de maestros.

\section{Reconocimiento del campo conceptual de la pedagogía}

Muchas veces en el discurso cotidiano las personas usan indistintamente los términos pedagogía, educación y didáctica. Sin embargo, es preciso establecer una precisión en aras de mostrar cómo la designación de estas aparentes sinonimias presenta, por el contrario, fronteras conceptuales producto de la existencia de un campo de producción teórica que ha establecido significados para estas palabras.

En pocas palabras, la pedagogía reflexiona sobre la educación y, consecuentemente, su propósito es generar saberes sobre la educación (Jaramillo Uribe, 1978; Lucio A., 1989). Dado que una de las prácticas presentes en el proceso educativo es la enseñanza, la pedagogía reflexiona sobre ésta y, para ello, construye conocimientos sobre la didáctica.

Lo anterior permite afirmar que la pedagogía es un campo conceptual, dado que en él se identifican objetos, conceptos y métodos (Zuluaga G., Echeverri, Martínez, Restrepo, \& Quiceno, 2011), y existen diferentes discursos y producciones teóricas al respecto.

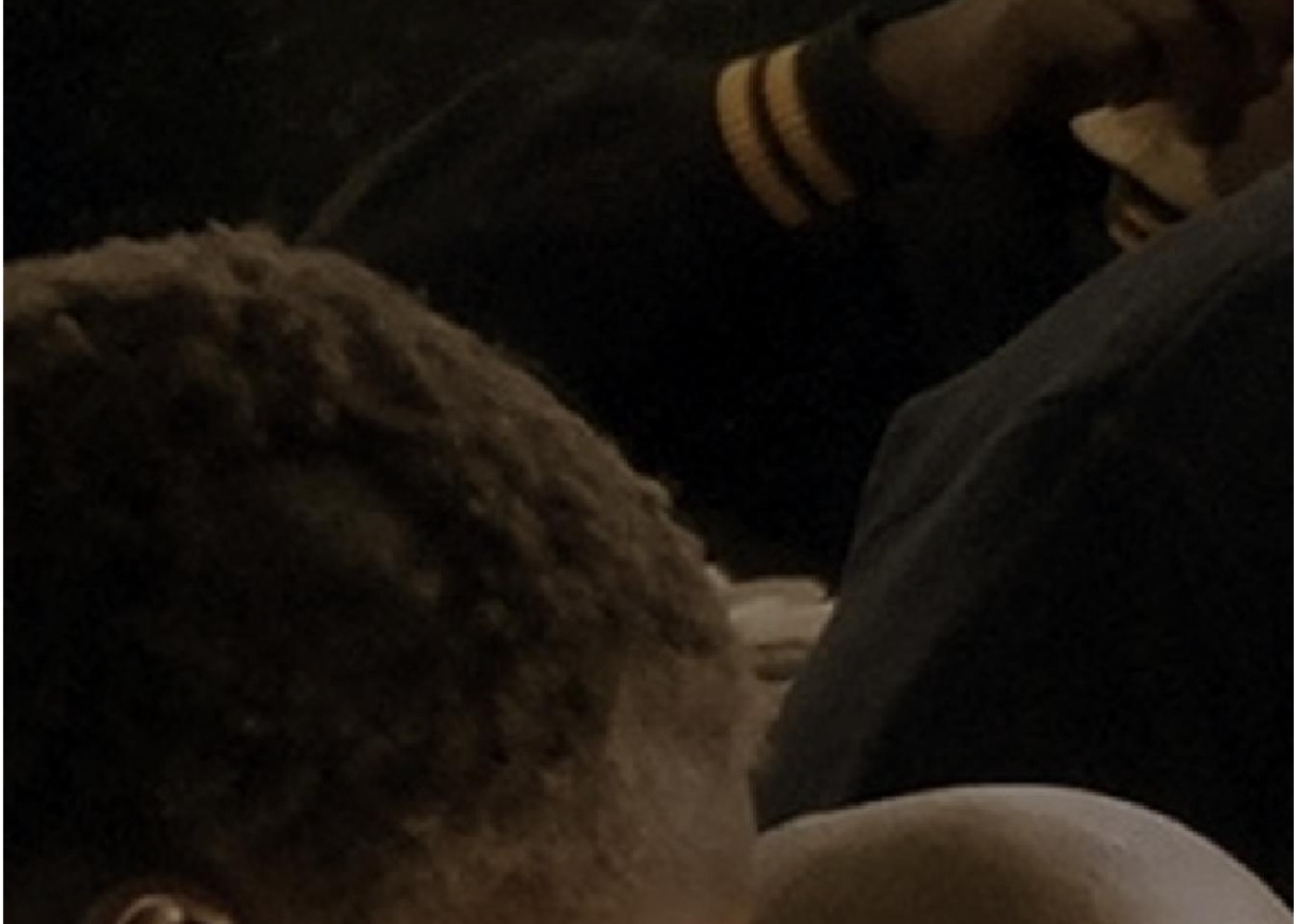


Incluso, al interior del campo se pueden ver pujas frente a estos objetos, conceptos y métodos, por ejemplo, para el grupo de Zuluaga G. y sus colaboradores (2011), la pedagogía conceptualiza, aplica, experimenta, conocimientos referentes a la enseñanza de los saberes en las diferentes culturas, diferente a Lucio A. (1989), quien afirma que la pedagogía piensa la educación. Además, podemos hablar de que existe un campo de la pedagogía porque éste hace posible que los maestros produzcan discursos sobre su saber. (Palacio Mejía, 2004)

No obstante, existen autores que plantean que el campo pedagógico está estructurado por la reproducción discursiva, es decir, que los agentes de este campo están regulados en sus prácticas discursivas. En efecto, para Díaz (1995), el campo pedagógico sería un campo subsumido a relaciones de poder que lo configuran. Esto ocurre, según Berstein (1998), porque el discurso pedagógico está constituido por el principio recontextualizador, "que se apropia, recoloca, recentra y relaciona selectivamente otros discursos para establecer su propio orden" (pág. 63). En ese sentido, este principio crea campos recontextualizadores, con reglas y agentes con funciones recontextualizadoras.

Los campos de recontextualización serían, por un lado, el campo de recontextualización oficial (el Estado y sus agentes) y, por otro lado, el campo de recontextualización pedagógico (pedagogos, libros de texto, etc.). Para Berstein (1998), el primero tiende a controlar el segundo. En ese orden de ideas, al ser el campo pedagógico un campo regido por el contexto recontextualizador, tendería a ser dominado por el campo de recontextualización oficial, determinando las disposiciones, prácticas y discursos de sus agentes. Por ello, Díaz (1995) propone el campo intelectual de la educación como un campo no subsumido al control discursivo y su consecuente reproducción. Sería, entonces, un campo de producción discursiva cuyos individuos serían productores discursivos y participarían en la distribución y circulación de estos discursos. Igualmente, al ser un campo existirían en éste pujas entre grupos de intelectuales por controlar las orientaciones discursivas.

En últimas, la distinción que hace Díaz (1995) del campo pedagógico y del campo intelectual de la educación, reivindica la producción discursiva de éste y encierra a aquel en la reproducción discursiva. Así, el intelectual del campo educativo tiene la capacidad de crear, discutir, modificar teorías y prácticas en la producción del discurso; por su parte, aquellos que pertenecen al campo pedagógico les queda la pugna por la recontextualización, que tiende a ser acaparada por el campo oficial.

Contrario a esta idea, Echeverry y Zuluaga G. (1997) reivindican el campo pedagógico ya que para ellos la pedagogía tiene una autonomía conceptual. Es decir, no estaría atada a una reproducción discursiva. Además, señalan que el campo intelectual de la educación no le reconoce existencia a la pedagogía más allá de la escuela y a la condición económica, social, y cultura que controla el discurso.

Para Echeverry y Zuluaga G. (1997), la pedagogía, en consecuencia, tendría relativa independencia de los campos de recontextualización, pues posee objetos, métodos y análisis, que le permiten producir discursos no necesariamente anclados a la estructura oficial. Asimismo, la pedagogía sería un campo en la medida que no hay una producción universal, sino que existen diversas producciones teóricas, esto indica la existencia de la intrínseca pugna propia del campo.

Por otro lado, reconocer la pedagogía como campo conceptual permite recuperar la capacidad de reflexión y producción teórica del maestro, contrario a las ciencias de la educación que, como señalan Zuluaga G. y sus colaboradores (2011), hacen aportes desdey para dichas ciencias, por ejemplo, la sociología de la educación a la sociología. Hablar de ciencias de la educación y no de campo de la pedagogía trae como consecuencia que el maestro pierda su capacidad de construir un saber propio sobre su práctica, pues las teorías serían producidas por los saberes de otras ciencias (sociología, psicología, administración, etc.) (Zuluaga G., Echeverri, Martínez, Restrepo, \& Quiceno, 2011)

En consecuencia, reconocer el campo de la pedagogía y, por extensión, su autonomía y capacidad de producción discursiva, es revalidar la capacidad de investigar y teorizar que tienen los maestros. Esto va más allá de las políticas públicas que hablan de la profesionalización y la excelencia docente, que lo conducen a ser reproductor de contenidos determinados en los campos de recontextualización, de los que los maestros son cada vez menos participantes, pues son orientados por personas especializadas de otras disciplinas. La excelencia docente no logra cobijar la capacidad que tiene el maestro de reflexionar y producir conceptos sobre la educación y la enseñanza, debido a que se centra en lograr profesionales con capacidad de instruir en ciertos contenidos para que estudiantes logren unos resultados en pruebas estandarizadas (de las que pocas veces los profesores participan en su diseño).

Igualmente, reconocer el campo pedagógico permite al maestro la posibilidad de reivindicarse como sujeto "capaz de pensarse a sí mismo" (Zuluaga G., Echeverri, Martínez, Restrepo, \& Quiceno, 2011), puesto que desde la lógica impuesta en la política oficial es pensado por otros, en su mayoría economistas. Esta pretensión del campo pedagógico y la elevación del maestro no necesariamente significa desconocer la existencia del campo intelectual de la educación, sugiere que es pertinente tener en cuenta que los intelectuales del campo de la educación pueden ser de otras disciplinas y no precisamente maestros.

De por sí la discusión abierta frente a la pedagogía y las ciencias de la educación, así como la del campo pedagógico y el campo intelectual de la educación, evidencian la existencia de un debate epistemológico, teórico y conceptual queabre las puertas a la reconfiguración del campo pedagógico, es decir, el debate aviva las condiciones que posibilitan su existencia. En todo caso, es importante seguir entendiéndolo "como casa, siempre abierta, del pensamiento y la experiencia pedagógica". (Echeverri \& Zuluaga G., 1997, pág. 18)

Si bien los maestros no son necesariamente productores de los saberes disciplinares que enseñan (no todos producen conocimientos de física, matemáticas, química, filosofía, etc.), sí tienen la capacidad de producir desde el campo conceptual de la pedagogía, es decir, de reflexionar, debatir y proponer categorías y teorías pedagógicas, pues lo intrínseco a su oficio y saber es la pedagogía.

Del mismo modo, los maestros pueden determinar qué enseñar, para qué enseñarlo y cómo enseñarlo. Si bien para ello toman contenidos construidos en los campos de producción científica, pueden ser agentes recontextualizadores sin que la recontextualización sea captada por la estructura oficial. En otras palabras, gracias a su saber pedagógico, los maestros tienen toda la capacidad de intervenir en el currículo, sin limitarse a ser reproductores de contenidos preestablecidos. Esto permite que el campo de recontextualización oficial no agote la posibilidad del campo de recontextualización pedagógica. De igual forma, que el campo intelectual de la educación no encubra al campo conceptual de la pedagogía.

En síntesis, argumentamos que es necesario reconocer el campo conceptual de la pedagogía como condición fundamental para reivindicar el saber de los maestros y, por extensión, su capacidad de investigar y producir conocimientos pedagógicos, esto es, sobre la educación, la didáctica y la enseñanza. Consecuentemente, formar maestros implica introducirlos en el campo conceptual de la pedagogía, es decir, que conozcan, debatan e investiguen desde categorías teóricas de los cuerpos teóricos de la pedagogía. 


\section{La formación de maestros desde el encuentro con el saber y el deseo de saber}

El reconocimiento del campo pedagógico permite suponer que los maestros, sujetos del saber pedagógico, pueden entrar a tomar posición en este campo. Del mismo modo, formar maestros implica introducirlos al campo de la pedagogía, lo que implica un encuentro con las metodologías, categorías y teorías producidas en la historia de este campo.

En suma, formar maestros es, entre otras cosas, llevarlos al encuentro con el saber pedagógico. Esto implica, como se planteó anteriormente, la capacidad de recontextualizar saberes producidos en otros campos, pero, de fondo, tener la capacidad de reflexionar sobre el qué, con quiénes, cómo, para qué de la educación y el qué, cómo, para qué de la enseñanza (lo propio de la didáctica).

Como se puede advertir, esta capacidad de reflexión se asocia a preguntas permanentes, pues sin interrogantes se difumina su capacidad de producción discursiva en el campo. En efecto, las tensiones del campo pueden estar motivadas por la insatisfacción frente a los discursos posicionados. Hablamos no sólo de una insatisfacción derivada de pugnas por el control, sino de una insatisfacción porque los discursos, valga decir, los conceptos y teorías, no explican satisfactoriamente la realidad educativa.

El campo es rico en discursos cuando los agentes no están satisfechos con el conocimiento producido, en otros términos, cuando los sujetos expresan sus dudas frente a lo producido, lo que es síntoma del deseo de saber. Así, sumergir al maestro en formación en el campo pedagógico implica motivar en él la sospecha por la explicación dada, la pregunta frente a lo producido teóricamente, pues, siguiendo a Bustamante (2013, pág. 270), "si el maestro se muestra sin dudas, entonces está dando a saber que no desea saber". Lo que sería deseable para el campo de recontextualización oficial, que espera de él la reproducción de contenidos, pero perverso para el campo pedagógico, que depende de su capacidad reflexiva.

Además, esto deriva en el reto de formar maestros que deseen saber para que puedan transmitir ese deseo cuando sean maestros, teniendo en cuenta que la escuela se basa en llevar al sujeto (estudiante) a un encuentro con el saber que produzca efectos en su subjetividad (Bustamante, 2013). Por demás, "el secreto del vínculo educativo es que el maestro mismo esté interesado" (Bustamante, 2013, pág. 309), es decir, el secreto no es un control escrupuloso en el diseño del currículo ni la implantación de metodologías exitosas en otros contextos.

Para sumergir al maestro en formación al campo pedagógico es necesario que él "conozca no sólo la situación actual de la pedagogía como los problemas que estructuran el discurso del cual es portador, sino también a sí mismo como sujeto de saber" (Palacio Mejía, 2004, pág. 121). Asimismo, como plantean Echeverry y Zuluaga G. (1997), los maestros que ingresan al campo precisan del diálogo entre el presente y pasado del campo, de la producción acumulada del campo.

Al mismo tiempo, el maestro en formación debe tener la inquietud de entretejer su saber pedagógico con los saberes con los que ha interactuado siempre la pedagogía, como las humanidades, las ciencias naturales, ciencias sociales, etc. (Echeverri \& Zuluaga G., 1997). El maestro, en consecuencia, tiene una doble necesidad de saber: la del campo pedagógico y la del campo disciplinar del que toma los contenidos de su enseñanza.
Este acercamiento a la producción acumulada en el campo, así como la compresión de sus tensiones, permitiría formar maestros que no se limiten a ofrecer discursos centrados en la instrucción, propios de la política de la excelencia docente, dado que "el discurso de instrucción está siempre integrado en el discurso regulador y éste es el discurso dominante” (Berstein, 1998, pág. 45). Lo anterior implica que el profesor en formación asuma un doble reto: sumergirse en el campo pedagógico y estar al tanto del campo de producción disciplinar (de donde extrae lo que enseña), lo que le permitiría tener herramientas para transgredir las lógicas de control del campo de recontextualización oficial. En efecto, si está más cercano al campo de producción tendrá más capacidad de agenciamiento en la recontextualización, pero si no será captado por el oficial.

Todo lo anterior implica un reto para las facultades que forman maestros, tanto a nivel de las licenciaturas como de posgrado, dado que implica en ellos despertar el deseo por el saber, tanto del campo pedagógico, como del campo disciplinar, lo que se logra, siguiendo a Bustamante (2013), con maestros universitarios que transmitan el deseo de saber y esto es, en últimas, inculcar la duda y la insatisfacción por los discursos del campo que se van convirtiendo en sentido común [diría Zemelman (2005), estar atentos a las teorías que van anquilosando la mirada y dan explicaciones automáticas a los hechos que presenta la realidad].

\section{Articulación de estos planteamientos con la formación de educadores en la Ley General de Educación}

La propuesta de formar maestros a partir de sumergirlos, mediante el deseo de saber, en el campo pedagógico e invitarlos a estar atentos al campo de producción disciplinar de los contenidos que enseñan, tiene alta relación con los lineamientos de la Ley General de Educación. (Ley 115 de 1994)

Efectivamente, en el artículo 109 de la Ley 115 de 1994 se lee que las finalidades de la formación de educadores son, entre otras, "Formar un educador de la más alta calidad científica y ética; Desarrollar la teoría y la práctica pedagógica como parte fundamental del saber del educador; Fortalecer la investigación en el campo pedagógico y en el saber específico".

Es decir, la ley se articula con las iniciativas que defienden el reconocimiento del campo pedagógico, dado que habla explícitamente de la teoría pedagógica como parte del saber del educadory, más aún, expresa la necesidad de fortalecer la investigación en este campo. En ese orden, lo estipulado en la Ley General de Educación reivindica el saber del maestro y su capacidad de reflexión frente al qué, cómo y para qué de su práctica. Lo que implica pensar al maestro no sólo como conocedor de un tema sino como investigador de su propia práctica. (Burgos Calderón \& Cifuentes Garzón, 2015)

Reconocer la existencia del campo pedagógico, como lo hace la Ley 115 de (1994), permite pensar una propuesta de formación de maestros efectuada desde el deseo de saber (lo que desemboca en el doble reto de acercarse tanto al campo pedagógico como al campo de producción disciplinar), tal como lo defendemos en este texto. 
Esta finalidad de formar educadores expuesta en la ley implica una diferencia sustancial con la noción de formar docentes, pues en los primeros se reconoce la reflexión por la enseñanza y en los segundos no (Álvarez Gallego, 2014). Además, educar educadores, finalidad estipulada en el capítulo 2 de la Ley 115 (1994), se realiza cuando se lleva al maestro en formación al encuentro con el campo pedagógico, lo que puede darse, según se ha defendido en este artículo, mediante el estímulo de un deseo de saber, que le permite al maestro construir conocimientos que lo reivindiquen como "sujeto de su saber”. (Saldarriaga Vélez, 2003, pág. 254)

Además, reivindicar al maestro como sujeto de saber permitiría darle una vigencia social que logré reconocerlo como profesional con cualidades específicas, que le permitirían construir una identidad diferencial (Charris Sandoval, Molano Caro, \& Torres Castillo, 2016), por encima de las estructuras que lo condicionan.

\section{Propuesta didáctica para que los estudiantes de las licenciaturas y posgrados en educación logren sumergirse en el campo conceptual de la pedagogía}

De acuerdo a los lineamientos indicados en la Ley, la misión de formar maestros está vinculada al reconocimiento del campo pedagógico y, consecuentemente, formarlos podría ser entendido como acercarlos al campo para que así adquieran conceptos y marcos teóricos que les permitan pensar, hablar, escribir y leer como pedagogos.

Este ejercicio de sumergirse en el campo pedagógico, a la par de acercarse al campo de producción disciplinar, implica pensar principios didácticos que coadyuven a lograr este propósito. Sin embargo, al ser la didáctica un área de reflexión del campo pedagógico, ésta no puede centrarse en criterios metodológicos sobre la enseñanza. Consecuentemente, es pertinente ver a "la enseñanza no sólo como práctica sino como objeto de análisis". (Zuluaga G., Echeverri, Martínez, Restrepo, \& Quiceno, 2011, pág. 32)

Dado que la propuesta en este documento es acercar al maestro en formación al acumulado teórico y conceptual del campo pedagógico despertándole el deseo de saber, el formador de maestros debería procurar la formulación de la duda permanente. Por consiguiente, el formador de maestros puede recurrir a una didáctica centrada en la duda, es decir, enseñar indagando y provocando la reflexión del qué enseñar, para qué hacerlo, a quiénes y cómo, pues una didáctica centrada únicamente en lo metodológico (cómo enseñar), da por sentado los contenidos y limita al maestro a ser un reproductor de dichos contenidos (más aún si son determinados en el campo de recontextualización oficial), restándole el estatuto intelectual al maestro y su capacidad de producir saberes.
La didáctica centrada en la duda permanente, que siempre tiene preguntas sobre la enseñanza, se entrelaza con el deseo de saber al no partir de contenidos dados, por el contrario, provoca una permanente "ruptura de sentidos y significados" (Quintar, 2006, pág. 41). En síntesis, es enseñar para la sospecha y la insatisfacción, cuestionando las verdades anquilosadas, lo producido como normal o natural, no solo en los sentidos comunes sino en las teorías que se van petrificando como verdades y eliminan el deseo de saber porque ya tienen respuesta previa a las preguntas (Cuesta Moreno, 2015). Así, el formador de formadores es un maestro "que mantiene al que busca en su rumbo, ese rumbo en el que cada uno está sólo en su búsqueda y en el que no deja de buscar". (Rancière, 2003, pág. 22)

Esta didáctica de la duda cimentada en el deseo de saber, que invita hacerle preguntas a los discursos producidos en el campo pedagógico y en el campo de producción disciplinar, abre la oportunidad de formar sujetos con capacidad de incidir en las realidades educativas, pues ellos tendrían elementos para comprender que son producto de la historia al mismo tiempo que productores de historia (Quintar, 2006). Lo que se traduce en "la pasión por enseñar en el encuentro de sujetos que deseen atreverse a aceptar el desafío de lo inédito". (Quintar, 2006, pág. 16)

En síntesis, hablamos de la formación de maestros que logren comprenderse como sujetos que se reconocen en permanente trámite (Bustamante, 2013), que no dan por sentado los sentidos que orientan su oficio y el papel de la educación, puesto que tienen preguntas permanentes que les permiten ser productores de saber. 


\section{Conclusión}

Este artículo proyectó la formación de maestros como un ejercicio de encuentro con el saber y, particularmente, por despertar el deseo de saber, de tal forma que se acerquen al campo conceptual de la pedagogía con dudas más que con certezas. Para lograrlo, es pertinente recurrir a una didáctica que provoque la sospecha, la duda, y no se anquilose en contenidos dados. Es pertinente decir que nada de esto tiene cabida si no se hace un reconocimiento del campo pedagógico. Por ello, lo propuesto en este escrito se articula con los lineamientos de la Ley General de Educación (Ley 115 de 1994).

Además, se puede concluir que una formación de maestros radica en que los estudiantes de licenciatura o de posgrados en educación deben sumergirse, a partir del deseo de saber, sumergirse en dos campos de producción: por un lado, el campo pedagógico y, por otro, el campo propio de la disciplina. El primero, le brinda las herramientas teóricas para pensar su práctica como maestro (fundamentalmente la enseñanza) y trabajador cultural; el segundo, le ofrece el acumulado de donde extrae lo que enseña.

Es pertinente decir que una reivindicación social del maestro reside en recuperar su relación con el saber. En efecto, ser maestro es demostrar una relación con el conocimiento que le permite profesar un saber (ser profesor), algo totalmente diferente a la política de capacitación docente, que encubre su relación con el saber para centrar su rol en ser un motivador del aprendizaje (por lo que ya no tiene que enseñar), incluso hay discursos que lo colocan como simple acompañante del aprendizaje, pues el estudiante aprende de manera autónoma.

Finalmente, lo anterior implicaría que los maestros en formación puedan tener espacios de discusión y reflexión sobre el significado y sentido de ser profesores hoy y, en ese orden, pensar las posibilidades de incidir en la configuración de otra perspectiva social y laboral a partir de lograr otra posición en el campo de producción pedagógica y en el campo de producción disciplinar.

\section{Referencias}

Álvarez Gallego, A. (2014). Los Límites de la Economía de la Educación: Abrir la mirada. Revista Colombiana de Educación(67), 19-46. doi:10.17227/0120391.67rce19.46

Berstein, B. (1998). Pedagogía, Control Simbólico e Identidad (239 ed.). Madrid [es]: Morata.

Burgos Calderón, D. B., \& Cifuentes Garzón, J. E. (2015). La Práctica Pedagógica Investigativa: Entre saberes, quereres y poderes. Horizontes Pedagógicos, 17(2), 118-127. Obtenido de http://revistas.iberoamericana. edu.co/index.php/rhpedagogicos/article/view/821

Bustamante, G. (2013). Sujeto, Sentido y Formación. Bogotá D.C. [co]: UPN, Universidad Pedagógica Nacional.

Charris Sandoval, J. E., Molano Caro, G., \& Torres Castillo, D. J. (2016). Caracterización de la Identidad Profesional de Educadores. Horizontes Pedagógicos, 18(1), 50-57. Obtenido de http://revistas.iberoamericana. edu.co/index.php/rhpedagogicos/article/view/1032

Cuesta Moreno, O. J. (2015). Educación, Presente y Potencia: La formación de conciencia epistémica a partir del encuentro con el otro. El Ágora USB, 15(1), 153-168. doi:10.21500/16578031.8

Díaz, M. (1995). Aproximaciones al Campo Intelectual de la Educación. En J. Larrosa (Ed.), Escuela, Poder y Subjetivación (pág. 366). Madrid [es] La Piqueta. Obtenido de http://wWw.fceia.unr.edu.ar/geii/maestria/ DoraBibliografia/Ut.\%201/Diaz.\%20campo\%20intelectual.pdf

Echeverri, A., \& Zuluaga G., O. L. (1997). Campo Intelectual y Campo Pedagógico de la Educación. Educación y Ciudad(4), 12-23.

Fundación Compartir. (2014). Tras la Excelencia Docente: Cómo mejorar la calidad de la educación para todos los colombianos. (L. Barragan Montaña, Ed.) Bogotá D.C. [Co]: Fundación Compartir.

Jaramillo Uribe, J. (1978). Historia de la Pedagogía como Historia de la Cultura (113 ed.). Bogotá D.C. [co]: Universidad de los Andes; CESO.

Ley 115 de 1994, Ley General de Educación (Congreso de la República de Colombia 8 de 2 de 1994). Obtenido de http://wwW. secretariasenado.gov. co/senado/basedoc/ley 0115 1994.html

Lucio A., R. (1989). Educación, Pedagogía, Enseñanza y Didáctica: Diferencias y relaciones. Revista de la Universidad de La Salle(17), 35-46. Obtenido de https://revistas.lasalle.edu.co/index.php//s/article/view/4712

Palacio Mejía, L. V. (2004). Elementos para Configurar un Dispositivo de Formación de Maestros. Educación y Pedagogía, XVI(40), 115-130. Obtenido de http://aprendeenlinea.udea.edu.co/revistas/index.php/revistaeyp/article/ view/6017

Quintar, E. B. (2006). La Enseñanza como Puente a la Vida (126 ed.). México D.F. $[\mathrm{mx}]$ : Instituto Politécnico Nacional.

Rancière, J. (2003). El Maestro Ignorante: Cinco lecciones sobre la emancipación intelectual (184 ed., Vol. 103 Laertes Educación). (N. Estrach Mira, Trad.) Barcelona [es]: Sedición.

Saldarriaga Vélez, O. d. (2003). Del Oficio del Maestro: Prácticas y teorías de la pedagogía moderna en Colombia (320 ed., Vol. 2). Bogotá D.C. [co]: Cooperativa Editorial Magisterio.

Zemelman, H. (2005). Voluntad de Conocer: El sujeto y su pensamiento en el paradigma crítico (Vols. 47 Autores, textos y temas: Ciencias sociales). Barcelona [es]: Anthropos; Universidad Autónoma de Chiapas.

Zuluaga G., O. L., Echeverri, A., Martínez, A., Restrepo, S., \& Quiceno, H. (2011). Educación y Pedagogía una Diferencia Necesaria. En O. L. Zuluaga G., Pedagogía y epistemología (págs. 21-40 [336]). Bogotá D.C. [co]: Cooperativa Editorial Magisterio. 